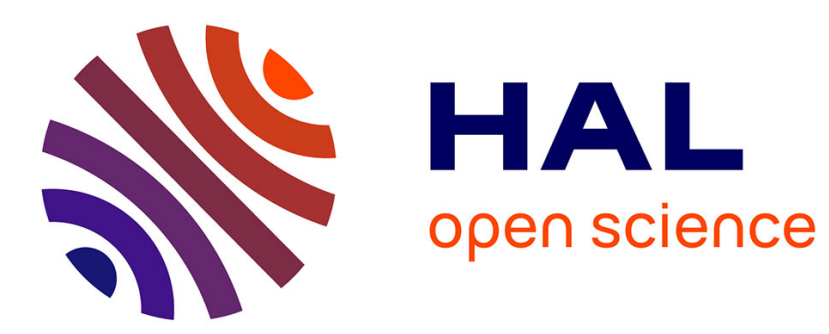

\title{
On the nonlinear interactions and existence of breathers in a chain of coupled pendulums
}

\author{
Alireza Ture Savadkoohi, Claude-Henri Lamarque, Jean David Goutte
}

\section{To cite this version:}

Alireza Ture Savadkoohi, Claude-Henri Lamarque, Jean David Goutte. On the nonlinear interactions and existence of breathers in a chain of coupled pendulums. International Journal of Non-Linear Mechanics, 2017, 94, pp.375-379. 10.1016/j.ijnonlinmec.2017.01.018 . hal-01463022

\section{HAL Id: hal-01463022 \\ https://hal.science/hal-01463022}

Submitted on 28 May 2020

HAL is a multi-disciplinary open access archive for the deposit and dissemination of scientific research documents, whether they are published or not. The documents may come from teaching and research institutions in France or abroad, or from public or private research centers.
L'archive ouverte pluridisciplinaire HAL, est destinée au dépôt et à la diffusion de documents scientifiques de niveau recherche, publiés ou non, émanant des établissements d'enseignement et de recherche français ou étrangers, des laboratoires publics ou privés. 


\title{
ON THE NONLINEAR INTERACTIONS AND EXISTENCE OF BREATHERS IN A CHAIN OF COUPLED PENDULUMS
}

\author{
A PREPRINT
}

\author{
A. Ture Savadkoohi* \\ Univ Lyon, ENTPE, LTDS UMR CNRS 5513 \\ Rue Maurice Audin F-69518 Vaulx-en-Velin Cedex, France \\ alireza.turesavadkoohi@entpe.fr \\ C.-H. Lamarque \\ Univ Lyon, ENTPE, LTDS UMR CNRS 5513 \\ Rue Maurice Audin, F-69518 Vaulx-en-Velin Cedex, France \\ lamarque@entpe.fr \\ J. D. Goutte \\ Univ Lyon, ENTPE \\ Rue Maurice Audin F-69518 Vaulx-en-Velin Cedex, France \\ jean-david.goutte@cerema.fr
}

\begin{abstract}
The paper considers a chain of linearly coupled pendulums. Continues first order system equations are treated via time and space multiple scale method which lead to nonlinear Schrödinger equation. Further investigations on the nonlinear Schrödinger equation detects systems responses in the form of propagated nonlinear waves as functions of their envelope and phases. This provides information about localization of nonlinear waves and their directions in space and time.
\end{abstract}

Keywords coupled pendulums $\cdot$ nonlinear Schrödinger equation $\cdot$ nonlinear waves $\cdot$ localization

\section{Introduction}

There are different ways of passively controlling and/or localizations of vibratory energies of main structural systems via coupled oscillators. The process can be categorized globally into two distinct groups: coupling linear (Frahm, 1911) and nonlinear (Ibrahim 2008) systems to principal structures. The localizer systems can be single linear or nonlinear oscillator (Hoang et al., 2016; Ture Savadkoohi et al., 2016) or a chain of oscillators with different types of coupling terms (Manevitch| 2001: Peyrard and Dauxois. 2004; Iooss and James, 2005; Manevitch et al., 2006; Gendelman et al. 2015: Hasan et al. 2015). In this paper, the localizer system is assumed to be a chain of pendulums which are attached to a rigid support from one end and are coupled to each other via linear springs from another end. We are interested to find out the possibility of localization of nonlinear waves in the chain as a function of time and space. This paper is a preliminary study to reach the following goal: controlling main structural systems against vibrations by localizing their energies into a chain of coupled pendulums. It should be underlined that the nonlinear energy localizations can be found also in many other nonlinear discrete and continuous oscillator systems, in addition to the chain of coupled pendulums studied in this paper (Vakakis et al., 1993, 1996; Manevitch and Smirnov, 2010; Smirnov et al., 2016, Strozzi et al. 2016). Organization of the paper is as it follows: governing equations of the discrete system are derived in Sect. 2 and then continuous approximated model is introduced. A multiple scale technique is implemented in Sect. 3 for tracing system behaviors at different scales of time and space. This method permits to treat nonlinear system equations (derived for continuous model) and to trace response of the system as function of velocities of its envelope

\footnotetext{
${ }^{*}$ Corresponding author
} 
and phase. A numerical example is provided in Sect. 4 showing the localization process in a continuous chain and its direction in the space and time. Finally, the paper is concluded in Sect. 5.

\section{The system under consideration}

Let us consider a chain of coupled pendulums in the orthogonal coordinates $\left(o, \vec{e}_{1}, \vec{e}_{2}, \vec{e}_{3}\right)$ as is illustrated in Fig. 11 All pendulums are situated on a rigid base with the distance of $d$ from each other and they possess the same mass as $m$. All masses are coupled to each other by springs with equal rigidity as $k$. All imposed forces to each mass $m_{n}$ can be summarized as:

where

$$
\left\{\begin{array}{l}
\vec{P}=-m g \vec{e}_{2} \\
\vec{F}_{n-1}=k\left(\|\left(\vec{u}_{n-1, n} \|-d\right) \frac{\vec{u}_{n-1, n}}{\left\|\vec{u}_{n-1, n}\right\|}\right. \\
\vec{F}_{n+1}=-k\left(\|\left(\vec{u}_{n, n+1} \|-d\right) \frac{\vec{u}_{n, n+1}}{\left\|\vec{u}_{n, n+1}\right\|}\right.
\end{array}\right.
$$

The lever arm of forces in Eq. 1 is defined as:

$$
\vec{u}_{n-1, n}=\left(l\left(\sin \left(\theta_{n}\right)-\sin \left(\theta_{n-1}\right)\right)+d\right) \vec{e}_{1}+l\left(\cos \left(\theta_{n}\right)-\cos \left(\theta_{n-1}\right)\right) \vec{e}_{2}
$$

$$
\vec{r}=l \sin \left(\theta_{n}\right) \vec{e}_{1}+l \cos \left(\theta_{n}\right) \vec{e}_{2}
$$

Moments of given forces in Eq. 1 are summarized as:

$$
\begin{gathered}
\vec{M}_{P}=\vec{r} \wedge \vec{P}=-m g l \sin \left(\theta_{n}\right) \vec{e}_{3} \\
\vec{M}_{F_{n-1}}=\vec{r} \wedge \vec{F}_{n-1} \\
\vec{M}_{F_{n+1}}=\vec{r} \wedge \vec{F}_{n+1}
\end{gathered}
$$

where $\wedge$ stands for the wedge product of two vectors. Let us define $J$ as the moment of inertia; we can write:

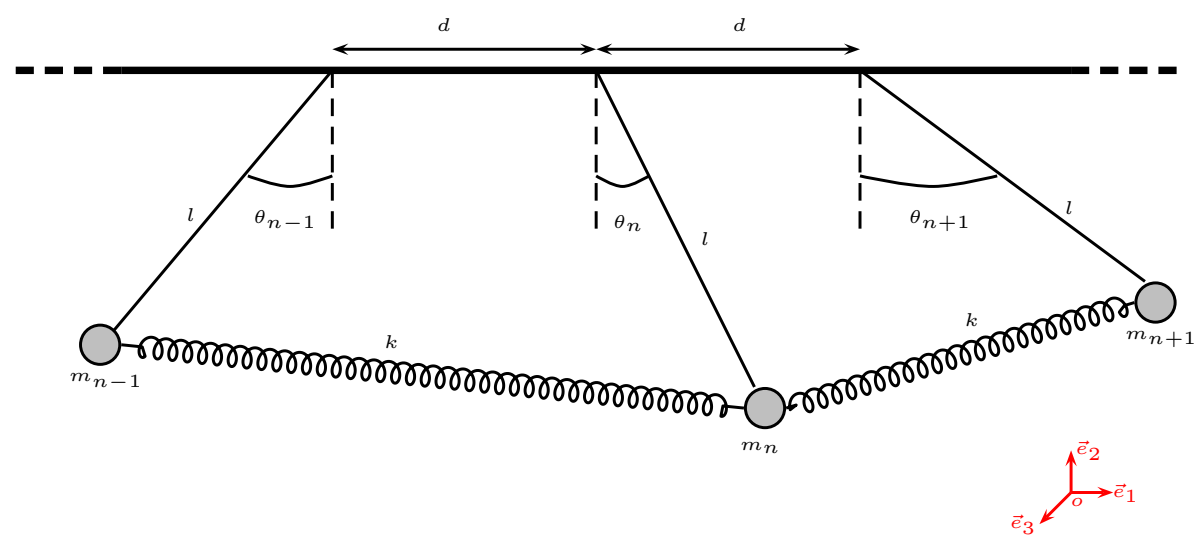

Figure 1: Schematic of the considered chain of coupled pendulums: All pendulums are equally spaced on a rigid base with the distance of $d$; they posses the same mass as $m\left(\cdots=m_{n-1}=m_{n}=m_{n+1}=\cdots=m\right)$ and they are coupled to each other by linear springs with equal rigidity as $k$.

$$
\ddot{\theta}_{n} J=-m g l \sin \left(\theta_{n}\right)+M_{F_{n-1}}+M_{F_{n+1}}
$$

Let us study $\vec{M}_{F_{n-1}}$ in detail (see Eq. 5. It reads:

$$
\begin{aligned}
& M_{F_{n-1}}=k\left(1-\frac{1}{\sqrt{1-2 \frac{l}{d}\left(\sin \left(\theta_{n}\right)-\sin \left(\theta_{n-1}\right)\right)-2 \frac{l^{2}}{d^{2}}\left(1-\cos \left(\theta_{n}-\theta_{n-1}\right)\right)}}\right) \\
& \times\left(-l d \cos \left(\theta_{n}\right)-l^{2} \sin \left(\theta_{n}-\theta_{n-1}\right)\right)
\end{aligned}
$$


Equation 8 can be re-written as:

$$
\begin{aligned}
& M_{F_{n-1}}=k\left(1-\left(1+\frac{l}{d}\left(\sin \left(\theta_{n}\right)-\sin \left(\theta_{n-1}\right)\right)\right.\right. \\
& \left.\left.+\frac{l^{2}}{d^{2}}\left(1-\cos \left(\theta_{n}-\theta_{n-1}\right)-\frac{3}{2}\left(\sin \left(\theta_{n}\right)-\sin \left(\theta_{n-1}\right)\right)^{2}\right)+\mathcal{O}\left(\frac{l^{3}}{d^{3}}\right)\right)\right) \\
& \times\left(-l d \cos \left(\theta_{n}\right)-l^{2} \sin \left(\theta_{n}-\theta_{n-1}\right)\right)
\end{aligned}
$$

We assume that $\theta \ll 2 \pi$. Equation 9 reads:

$$
\begin{aligned}
& M_{F_{n-1}}=k\left(-\frac{l}{d}\left(\theta_{n}-\theta_{n-1}\right)-\frac{l^{2}}{d^{2}}\left(\left(\theta_{n}-\theta_{n-1}\right)^{2}-\frac{3}{2}\left(\theta_{n}-\theta_{n-1}\right)^{2}\right)\right) \\
& \times\left(-l d-l^{2}\left(\theta_{n}-\theta_{n-1}\right)\right)
\end{aligned}
$$

For further developments we will introduce following new variables: $l=L d$ and $k=K \frac{d}{l}$. First order terms of Eq. 10 yield to

$$
M_{F_{n-1}}=-K L d^{2}\left(\theta_{n}-\theta_{n-1}\right)
$$

and with the same manner it can be demonstrated that

$$
M_{F_{n+1}}=K L d^{2}\left(\theta_{n+1}-\theta_{n}\right)
$$

Equation 7 reads

$$
\ddot{\theta}_{n} J=-m g L d \sin \left(\theta_{n}\right)+K L d^{2}\left(\theta_{n-1}-2 \theta_{n}+\theta_{n+1}\right)
$$

The Taylor expansion of Eq. 13 with respect to $\theta_{n}$ yields to

$$
\theta_{t t} J=-m g L d \sin (\theta)+K L d^{2} \theta_{x x}
$$

It should be mentioned that we did not expand the term of Eq. 4 intentionally for having similar equations of KleinGordon as is given in Eq. 14. If one keeps higher order terms of the Eq. 10, then following continuous system can will be obtained:

$$
\begin{array}{r}
\theta_{t t} J=-m g L d\left(\theta-\frac{\theta^{3}}{3}\right)+K L d^{4} \theta_{x x}-2 K L^{2} d^{4} \theta_{x}^{2} \theta-K L d^{4} \theta^{2} \theta_{x x} \\
-\frac{1}{6} K L d^{5} \theta_{x}^{3}+6 K L^{2} d^{5} \theta_{x} \theta_{x x}-\frac{5}{36} K L d^{5} \theta_{x x x}-\frac{16}{3} K L^{2} d^{5} \theta_{x}^{3} \theta-K L d^{5} \theta \theta_{x} \theta_{x x}
\end{array}
$$

It should be underlined that Rosenau (1986, 1987), studied the dynamics of mass-spring chains with arbitrary interparticle and substrate potentials by describing an approach for deriving governing system equations near continuum limits. His explained method captures all terms to given order in discreteness and allows to express well-posed nonlinear partial differential equations for such systems. However, comparison between obtained higher order differential equation in this paper which is described in Eq. 14 with the explained methodology of Rosenau (1986, 1987) is beyond the scope of the current paper.

In the current paper we will investigate on the Eq. 14. Apart from the system under study of this paper, Eq. 14 governs several physical and mechanical systems such as Josephson junction transmission line, a series of pendulums connected by a spring at base, motion of a Bloch wall between ferromagnetic domain, motion of a slide dislocation in a crystalline structure, Lorentz contraction, etc. (Scott, 1969). Localized solutions of the Sine-Gordon equation have been studied by Kochendörfer and Seeger (1950,1951). Scott (1969) looked for the solution of the Eq. 14 as waves of permanent profile while properties of Sine-Gordon equation have been described by Ablowitz et al. (1973) and Zakharov et al. (1984).

\section{Tracing system behaviors at different scales of time and space}

To detect system behavior, we are interested to endow a multi-scale method (Nayfeh and Mook, 1979; Dodd et al. 1982, Peyrard and Dauxois, 2004, Ostrovsky, 2015). We introduce a very small parameter $0<\epsilon \ll 1$ which connects different scales of time and space as $T_{\alpha}=\epsilon^{\alpha} t, X_{\alpha}=\epsilon^{\alpha} x, \alpha \in \mathbb{N}$, respectively. We set

$$
\theta(x, t)=\epsilon \sum_{\alpha=0}^{n} \epsilon^{\alpha} \phi_{\alpha}\left(X_{0}, X_{1}, \ldots, T_{0}, T_{1}, \ldots\right)
$$


In this case derivatives with respective to time $t$ and the space $x$ yield to

$$
\begin{gathered}
\frac{d}{d t}=\frac{d T_{0}}{d t} \frac{\partial}{\partial T_{0}}+\frac{d T_{1}}{d t} \frac{\partial}{\partial T_{1}}=D_{0}+\epsilon D_{1}+\ldots \\
\frac{d^{2}}{d t^{2}}=D_{0}^{2}+2 \epsilon D_{0} D_{1}+\epsilon^{2}\left(D_{1}^{2}+2 D_{0} D_{2}\right)+\ldots \\
\frac{d}{d x}=D_{X 0}+\epsilon D_{X 1}+\ldots \\
\frac{d^{2}}{d x^{2}}=D_{X 0}^{2}+2 \epsilon D_{X 0} D_{X 1}+\epsilon^{2}\left(D_{X 1}^{2}+2 D_{X 0} D_{X 2}\right)+\ldots
\end{gathered}
$$

Since we are interested to detect the behavior of the system described in Eq. 14, we will study this equation at different orders of $\epsilon$ in following sections.

\subsection{System behavior at the order of $\epsilon^{1}$}

Equation 14 at $\epsilon^{1}$ order reads

$$
J D_{0}^{2} \phi_{0}=-m g L d \phi_{0}+K L d^{2} D_{X 0}^{2} \phi_{0}
$$

We introduce an operator $\hat{L}$. Equation 21 yields to:

$$
\left(J D_{0}^{2}+m g L d-K L d^{2} D_{X 0}^{2}\right) \phi_{0}=\hat{L} \phi_{0}=0
$$

This equation illustrates that $\phi_{0}$ is a wave with following definitions:

$$
\phi_{0}(x, t)=A\left(X_{1}, X_{2}, \ldots, T_{1}, T_{2}, \ldots\right) e^{i\left(q X_{0}-\omega T_{0}\right)}+c . c .
$$

where $c_{0}^{2}=\frac{K L d^{2}}{J}, \omega_{0}^{2}=\frac{m g L d}{J}$ and $\omega^{2}=\omega_{o}^{2}+c_{0}^{2} q^{2}$.

\subsection{System behavior at the order of $\epsilon^{2}$}

Equation 14 at the $\epsilon^{2}$ order reads:

$$
\hat{L} \phi_{1}=\left(-2 D_{0} D_{1}+2 c_{0}^{2} D_{X 0} D_{X 1}\right) \phi_{0}
$$

or

$$
\hat{L} \phi_{1}=\left(2 i \omega A_{T 1}+2 i q c_{0}^{2} A_{X 1}\right) e^{i\left(q X_{0}-\omega T_{0}\right)}
$$

If $e^{i\left(q X_{0}-\omega T_{0}\right)} \subset \operatorname{Ker}(\hat{L})$, then

$$
\frac{\partial A}{\partial T_{1}}+\frac{q c_{0}^{2}}{\omega} \frac{\partial A}{\partial X_{1}}=0
$$

The characteristic curve of Eq. 26 reads as:

$$
X_{1}-\frac{q c_{0}^{2}}{\omega} T_{1}=\mathfrak{C} \in \mathbb{R}
$$

So, the amplitude $A$ in Eq. 26 can be represented as

$$
A\left(X_{1}, T_{1}, X_{2}, T_{2}, \ldots\right)=A\left(X_{1}-\frac{q c_{0}^{2}}{\omega} T_{1}, X_{2}, T_{2}, \ldots\right)
$$

\subsection{System behavior at the order of $\epsilon^{3}$ and detection of the response}

$\epsilon^{3}$ order of Eq. 14 yields to

$$
\begin{aligned}
& \hat{L} \phi_{2}=\left(-2 D_{0} D_{1}+2 c_{0}^{2} D_{X 0} D_{X 1}\right) \phi_{1}+\frac{\omega_{0}^{2}}{6} \phi_{0}^{3} \\
& +\left(-D_{1}^{2}-2 D_{2} D_{0}+c_{0}^{2}\left(D_{X 1}^{2}+2 D_{X 0} D_{X 2}\right)\right) \phi_{0}
\end{aligned}
$$

After removing secular terms of Eq. 29 we will have:

$$
-\frac{\partial^{2} A}{\partial T_{1}^{2}}+2 i \omega \frac{\partial A}{\partial T_{2}}+c_{0}^{2}\left(\frac{\partial^{2} A}{\partial X_{1}^{2}}+2 i q \frac{\partial A}{\partial X_{2}}\right)+\frac{\omega_{0}^{2}}{2}|A|^{2} A=0
$$


We introduce new variables as $\xi_{\alpha}=X_{\alpha}-v T_{\alpha}$ and $\tau_{\alpha}=T_{\alpha}$. so

$$
\begin{gathered}
\frac{\partial}{\partial T_{\alpha}}=\frac{\partial}{\partial \tau_{\alpha}}-v \frac{\partial}{\partial \xi_{\alpha}} \\
\frac{\partial^{2}}{\partial T_{\alpha}^{2}}=-v\left(\frac{\partial^{2}}{\partial \xi_{\alpha} \partial \tau_{\alpha}}-v \frac{\partial^{2}}{\partial \xi_{\alpha}^{2}}\right)+\frac{\partial^{2}}{\partial \tau_{\alpha}^{2}}-v \frac{\partial^{2}}{\partial \xi_{\alpha} \partial \tau_{\alpha}} \\
\frac{\partial}{\partial X_{\alpha}}=\frac{\partial}{\partial \xi_{\alpha}} \\
\frac{\partial^{2}}{\partial X_{\alpha}^{2}}=\frac{\partial^{2}}{\partial \xi_{\alpha}^{2}}
\end{gathered}
$$

Equation 30 reads:

$$
\begin{aligned}
& \left(c_{o}^{2}-v^{2}\right) A_{\xi_{1} \xi_{1}}-A_{\tau_{1} \tau_{1}}+2 v A_{\xi_{1} \tau_{1}}+\left(2 i c_{o}^{2} q-2 i \omega v\right) A_{\xi_{2}}+2 i \omega A_{\tau_{2}}+ \\
& \frac{\omega_{0}^{2}}{2}|A|^{2} A=0
\end{aligned}
$$

Considering new variables in Eq. 26 one can see that $A_{\tau_{1}}=0$ and $v=\frac{c_{0}^{2} q}{w}$. Then, Eq. 35 reads

$$
\frac{\left(c_{o}^{2}-v^{2}\right)}{2 \omega} A_{\xi_{1} \xi_{1}}+i A_{\tau_{2}}+\frac{\omega_{0}^{2}}{2 \omega}|A|^{2} A=0
$$

which is a nonlinear Schrödinger equation.

The solutions of this equation have been described in many references such as in (Dodd et al., 1982, Peyrard and Dauxois, 2004; Scott, 2005).

We introduce variables $P=\frac{c_{o}^{2}-v^{2}}{2 \omega}=\frac{c_{o}^{2} \omega_{0}^{2}}{2 \omega^{3}}$ and $Q=\frac{\omega_{0}^{2}}{2 \omega}$ which are strictly positive. Let as inject $A=$ $B\left(\xi_{1}, \tau_{2}\right) e^{i \delta\left(\xi_{1}, \tau_{2}\right)}$ in Eq. 36 , we reach to

$$
\begin{aligned}
& i\left(B_{\tau_{2}} e^{i \delta}+i B \theta_{\tau_{2}} e^{i \delta}\right)+P\left(B_{\xi_{1} \xi_{1}} e^{i \delta}+2 i B_{\xi_{1}} \delta_{\xi_{1}} e^{i \delta}-B\left(\delta_{\xi_{1}}\right)^{2} e^{i \delta}+i B \delta_{\xi_{1} \xi_{1}} e^{i \delta}\right) \\
& +Q|B|^{2} B e^{i \delta}=0
\end{aligned}
$$

Separation of real and imaginary parts of Eq. 37 provides:

$$
\begin{gathered}
B_{\tau_{2}}+2 P B_{\xi_{1}} \delta_{\xi_{1}}+P B\left(\delta_{\xi_{1} \xi_{1}}\right)=0 \\
-B \delta_{\tau_{2}}+P B_{\xi_{1} \xi_{1}}-P B\left(\delta_{\xi_{1}}\right)^{2}+Q|B|^{2} B=0
\end{gathered}
$$

We are interested in a solution where the phase $\delta$ propagates with the velocity of $u_{p}$ and the envelope $B$ propagates with the velocity of $u_{e}$ by keeping their own form. This means that we are searching for a solution in the following form:

$$
\left\{\begin{array}{l}
B\left(\xi_{1}, \tau_{2}\right)=B\left(\xi_{1}-u_{e} \tau_{2}\right) \\
\delta\left(\xi_{1}, \tau_{2}\right)=\delta\left(\xi_{1}-u_{p} \tau_{2}\right)
\end{array}\right.
$$

Here, there is a difference of order of $\epsilon$ between $\xi_{1}$ and $\tau_{2}$ (see Eq. 40. So, velocities of propagation should be $\mathcal{O}\left(\epsilon^{-1}\right.$ ). Equations 38 and 39 take following forms:

$$
\begin{gathered}
-u_{e} B_{x}+2 P B_{x} \delta_{x}+P B \delta_{x x}=0 \\
u_{p} B \delta_{x}+P B_{x x}-P B\left(\delta_{x}\right)^{2}+Q B^{3}=0
\end{gathered}
$$

We search for localized solitons of nonlinear Schrödinger system in space. This can be reached if $|x| \rightarrow \infty$, then $B \rightarrow 0$ and $B_{x} \rightarrow 0$. In this case, Eq. 41 provides

$$
\delta_{x}=\frac{u_{e}}{2 P}
$$

or

$$
\delta\left(\xi_{1}, \tau_{2}\right)=\frac{u_{e}}{2 P}\left(\xi_{1}-u_{p} \tau_{2}\right)
$$


Considering the solution of Eq. 44 in Eq. 42, we have

$$
\frac{u_{e} u_{p}}{2 P} B+P B_{x x}-\frac{u_{e}^{2}}{4 P} B+Q B^{3}=0
$$

Multiplying Eq. 45 by $P B_{x}$ and taking its integral, we reach to

$$
-\frac{u_{e}^{2}-2 u_{e} u_{p}}{8} B^{2}+\frac{P^{2}}{2} B_{x}^{2}+\frac{P Q}{4} B^{4}=0
$$

If we assume that $u_{e}>u_{p}$, the solution of the Eq. 46 reads

or

$$
B=\sqrt{\frac{u_{e}^{2}-2 u_{e} u_{p}}{2 P Q}} \operatorname{sech}\left(\sqrt{\frac{Q}{2 P}} \sqrt{\frac{u_{e}^{2}-2 u_{e} u_{p}}{2 P Q}}\left(\xi_{1}-u_{e} \tau_{2}\right)\right)
$$

$$
B=\sqrt{\frac{u_{e}^{2}-2 u_{e} u_{p}}{2 P Q}} \operatorname{sech}\left(\sqrt{\frac{Q}{2 P}} \sqrt{\frac{u_{e}^{2}-2 u_{e} u_{p}}{2 P Q}}\left(\epsilon x-\left(u_{e} \epsilon+v\right) \epsilon t\right)\right)
$$

and considering Eq. 44, $\theta(x, t)$ can be formulated as:

$$
\theta(x, t)=2 \epsilon B \cos \left(\frac{u_{e}}{2 P}\left(\epsilon x-\left(v+\epsilon u_{p}\right) t \epsilon\right)+(q x-\omega t)\right)
$$

The direction of propagation can be traced via Eq. 47 . It reads

$$
\xi_{0}-\epsilon u_{e} t=0
$$

or

$$
x-\left(v+\epsilon u_{e} t\right)=0
$$

and considering Eq. 49 the frequency of the propagation, $\omega_{p}$, on the propagation line can be defined as:

$$
\omega_{p}=\frac{\epsilon^{2} u_{e}}{2 P}\left(u_{e}-u_{p}\right)+q\left(v+\epsilon u_{e}-\frac{\omega}{q}\right)
$$

\subsection{A supplementary note in tracing the response of the system without $\epsilon$}

We can also trace the system behavior without having information about $\epsilon$ parameter. The Schrödinger equation (see Eq. 36. can be re-written as:

Let us assume that $\mathfrak{A}=\epsilon A$. Equation 53 yields to

$$
P A_{\xi_{0} \xi_{0}}+i A_{\tau_{0}}+\epsilon^{2} Q|A|^{2} A=0
$$

$$
P \mathfrak{A}_{\xi_{0} \xi_{0}}+i \mathfrak{A}_{\tau_{0}}+Q|\mathfrak{A}|^{2} \mathfrak{A}=0
$$

As previous steps, we can assume that $\mathfrak{A}=\mathfrak{B}\left(\xi_{1}, \tau_{2}\right) e^{i \hat{\delta}\left(\xi_{1}, \tau_{2}\right)}$ and then we search for a solution where the phase and the amplitude propagate with the velocities as $\hat{u}_{p}$ and $\hat{u}_{e}$ in the following form

$$
\left\{\begin{array}{l}
\mathfrak{B}\left(\xi_{1}, \tau_{2}\right)=\mathfrak{B}\left(\xi_{0}-\hat{u}_{e} \tau_{0}\right) \\
\hat{\delta}\left(\xi_{1}, \tau_{2}\right)=\hat{\delta}\left(\xi_{0}-\hat{u}_{p} \tau_{0}\right)
\end{array}\right.
$$

where $\hat{u}_{e}$ and $\hat{u}_{p}$ are at $\mathcal{O}(1)$. We obtain

$$
\begin{aligned}
& \theta(x, t)=2 \sqrt{\frac{\hat{u}_{e}^{2}-\hat{u}_{e} \hat{u}_{p}}{2 P Q}} \operatorname{sech}\left(\sqrt{\frac{Q}{2 P}} \sqrt{\frac{\hat{u}_{e}^{2}-\hat{u}_{e} \hat{u}_{p}}{2 P Q}}\left(x-\left(\hat{u}_{e}+v\right) t\right)\right) \times \\
& \cos \left(\frac{\hat{u}_{e}}{2 P}\left(x-\left(v+\hat{u}_{p}\right) t\right)+(q x-\omega t)\right)
\end{aligned}
$$

\section{An example}

For demonstrating the results in a given numerical example, we assume system parameters which are provided in Table 1. Variations of the amplitude $B$ with respect to space and time, obtained from Eq. 48, are illustrated in Fig. 22. It is seen that the amplitude of the system propagates in space and time in a specific direction. The two-dimensional view of this propagation which is presented in Fig. 3 shows that the amplitude propagates in the propagation direction which has been already predicted in Eq. 50. The three-dimensional view of propagation of $\theta$ obtained from Eq. 49 , is provided in Fig. 4 which is zoomed in Fig. 5 for small intervals of time and space. Variation of $\theta$ on the propagation direction (see Eq. 50p is illustrated in Fig. 6 6 showing that the system propagates with the frequency of $\omega_{p}=22.2150 \mathrm{~Hz}$ which can be evaluated via Eq. 52 . 


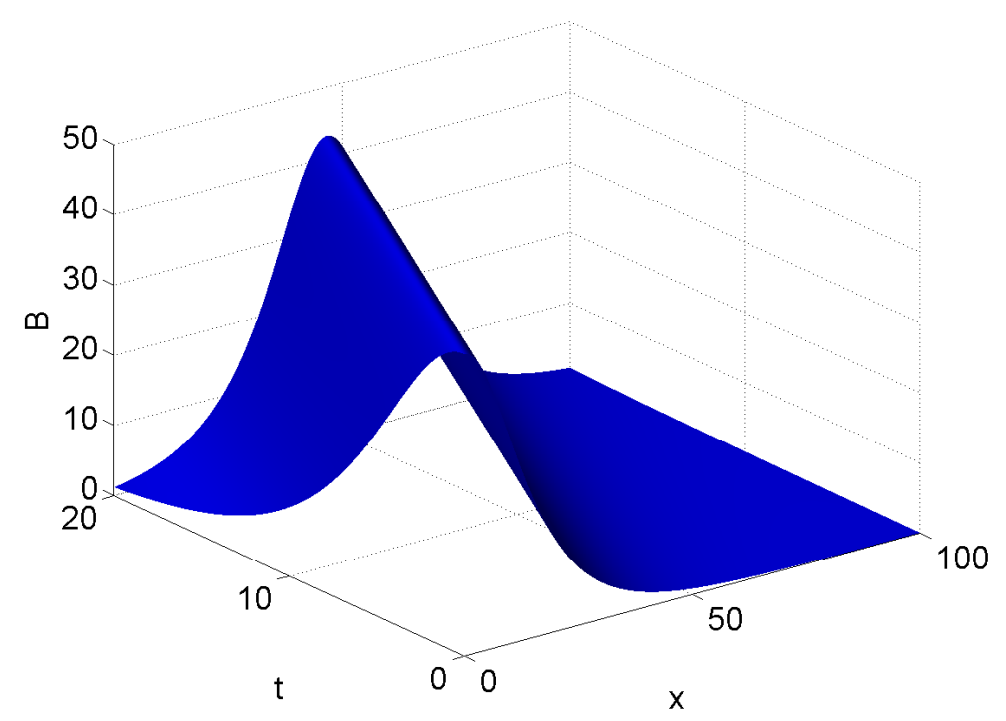

Figure 2: Obtained numerical results, $B$ versus $x$ and $t$, for given system parameters which are defined Table 1. Results are obtained via Eq. 48 for $x \in[0,100]$ and $t \in[0,20]$.

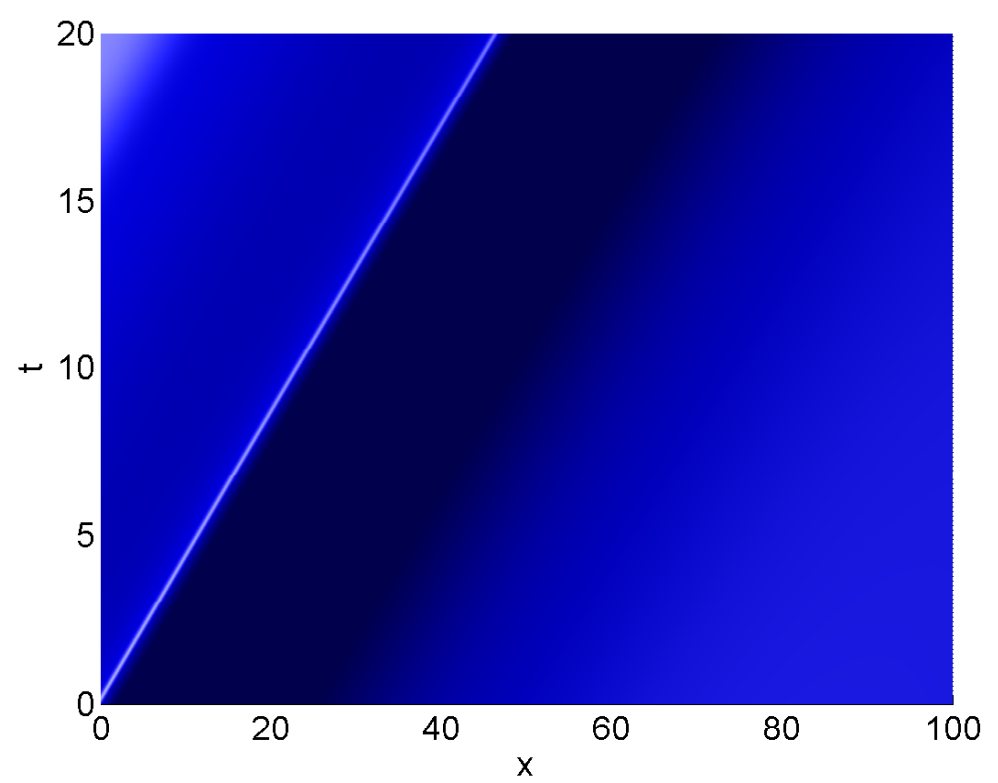

Figure 3: Two-dimensional view of Fig. 2, which presents the propagation direction. This direction can also be traced by Eq. 51 


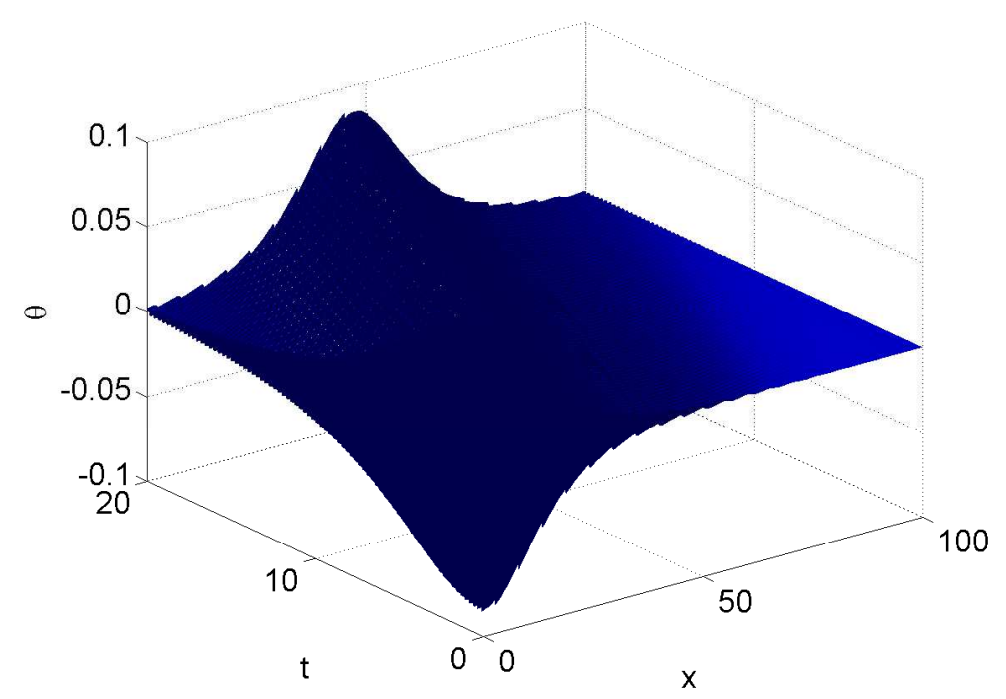

Figure 4: Obtained numerical results, $\theta$ versus $x$ and $t$, for given system parameters which are defined Table 1 . Results are obtained via Eq. 49 for $x \in[0,100]$ and $t \in[0,20]$.

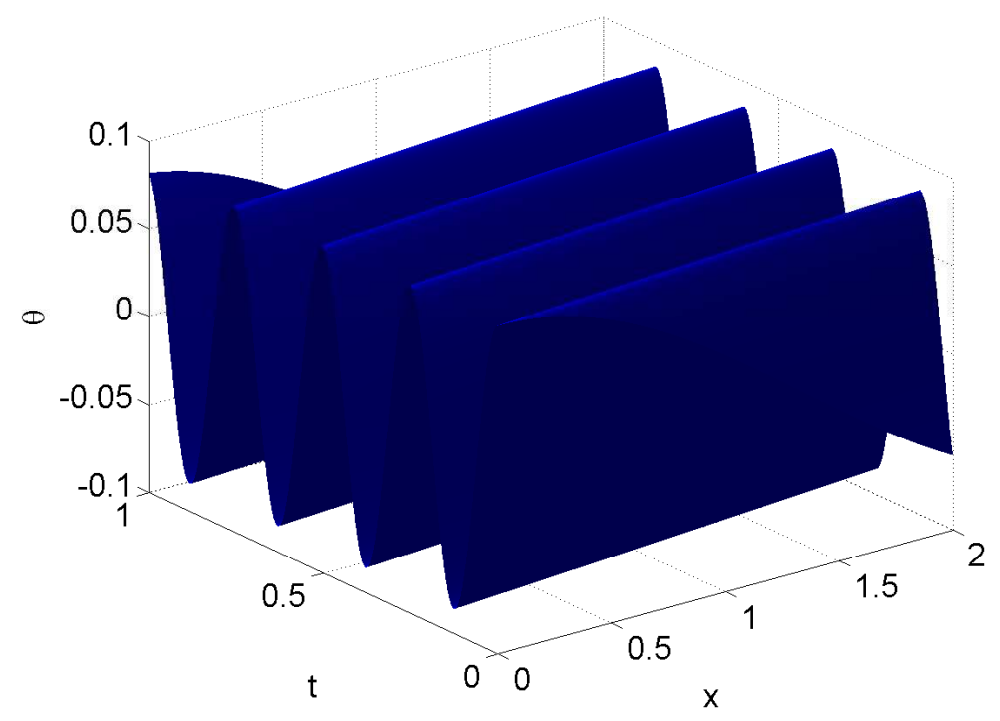

Figure 5: Obtained numerical results, $\theta$ versus $x$ and $t$, for given system parameters which are defined Table 1 . Results are obtained via Eq. 49 for $x \in[0,2]$ and $t \in[0,1]$. 


\begin{tabular}{|c|c|c|c|c|c|c|c|c|}
\hline$k$ & $d$ & $l$ & $g$ & $m$ & $q$ & $\epsilon$ & $\epsilon u_{e}$ & $\epsilon u_{p}$ \\
\hline 0.5 & 0.01 & 0.0177 & 9.81 & 0.01 & 1 & $10^{-3}$ & 0.3 & 0.1 \\
\hline
\end{tabular}

Table 1: Numerical values for system parameters.

(a)

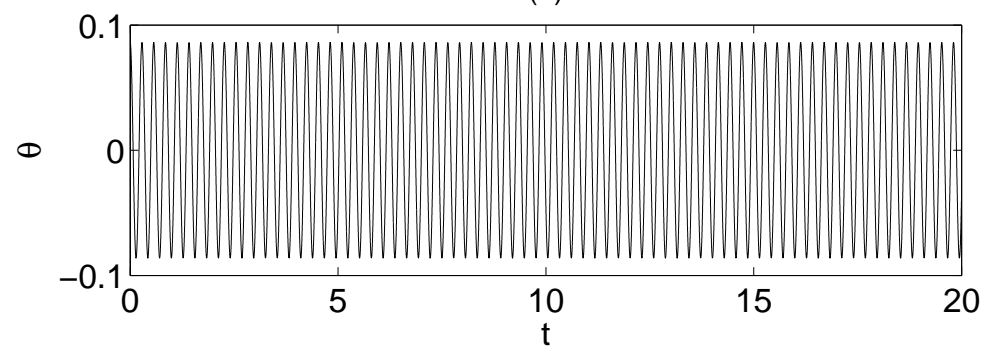

(b)

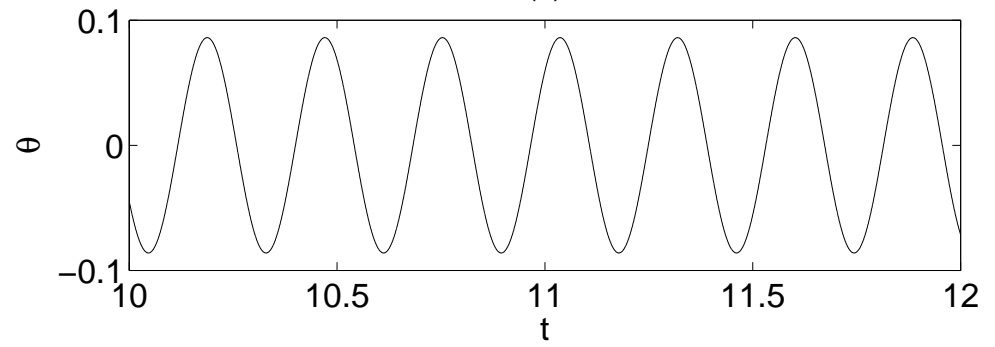

Figure 6: Variation of $\theta$ on the propagation direction which is defined in Eq. 51 during two time intervals: a) $t \in[0,20]$ ; b) $t \in[10,12]$. The frequency of the propagation can be obtained via Eq. 52 It provides $\omega_{p}=22.2150 \mathrm{~Hz}$ which is in agreements with the frequency of propagation in this figure.

\section{Conclusions}

Dynamics and nonlinear wave propagation in a chain of pendulums is studied: pendulums are equally spaced on a rigid base and are connected to each other from other side via linear springs. To trace behaviors of the continuous system, a multiple scale technique is implemented which results on the emerge of nonlinear Schrödinger equations. Searching for localized solitons of nonlinear Schrödinger system in space, leads to detection of phase and envelope of the propagation as functions of their velocities which provide also detection of propagation direction and frequency. Obtained information furnishes design tools for tuning system parameters for having appropriate propagation in terms of amplitude, phase, direction and frequency. As a perspective of current work, direct numerical integration can be carried out for a discrete chain of oscillators which contains a large number of pendulums. Then, parameters of localized waves of corresponding continuous system can be identified from numerical results for having comparison between two types of results. The studied chain can be used as the passive controller system for protection of principal structures and/or as the energy harvester.

\section{Acknowledgments}

The authors would like to thank the "Ministère de l'Environnement, de l'Energie et de la Mer" for supporting this work.

\section{References}

Ablowitz M. J., Kaup D. J., Newell A. C., Segur H., 1973. Method for Solving the Sine-Gordon Equation, Physical Review Letters 30, 1262-1264.

Dodd R. K.,Eilbeck J. C., Gibbon J. D., 1982. Solitons and nonlinear wave equations, London: Academic Press, 1982.

Frahm H., 1911 Device for damping vibrations of bodies, US Patent 989,958.

Gendelman O. V., Zolotarevskiy V., Savin A. V., Bergman L. A., Vakakis A. F., 2015. Accelerating oscillatory fronts in a nonlinear sonic vacuum with strong nonlocal effects, Physical Review E 93, 032216. 
Hasan M. A., Pichler L., Starosvetsky Y., McFarland D. M., Vakakis A. F., 2015. Effects of uncertainties on pulse attenuation in dimer granular chains with and without pre-compression, Continuum Mechanics and Thermodynamics 27, 749-766.

Hoang T., Ducharmea K., Kima Y., Okumusb P., 2016. Structural impact mitigation of bridge piers using tuned mass damper, Engineering Structures 112, 287-294.

Ibrahim R. A., 2008. Recent advances in nonlinear passive vibration isolators, Journal of Sound and Vibration 314, $371-452$.

Iooss G., James G., 2005. Localized waves in nonlinear oscillator chains, Chaos 25, 015113.

Kochendörfer A., Seeger A., 1950. Theorie der Versetzungen in eindimensionalen Atomreihen, Zeitschrift für Physik 127, 533-550.

Kochendörfer A., Seeger A., 1951. Theorie der Versetzungen in eindimensionalen Atomreihen: II. Beliebig angeordnete und beschleunigte Versetzungen, Zeitschrift für Physik 130, 321-336.

Manevitch L. I., 2001. The description of localized normal modes in a chain of nonlinear coupled oscillators using complex variables, Nonlinear Dynamics 25, 95-109.

Manevitch L. I., Savin A. V., Lamarque C.-H., 2006. Analytical study and computer simulation of discrete optical breathers in a zigzag chain, Physical Review B : Condensed matter and materials physics 74, 014305.

Manevitch L. I., Smirnov V. V., 2010. Limiting phase trajectories and the origin of energy localization in nonlinear oscillatory chains, Physical Review E 82, 036602.

Nayfeh A. H., Mook D. T., 1979. Nonlinear oscillations, John Wiley and Sons, 704.

Ostrovsky L., 2015. Asymptotic perturbation theory of waves, Imperial College Press, World Scientific Publishing Co. Pte.Ltd., 208.

Peyrard M., Dauxois T., 2004. Physique des solitons, EDP Sciences et CNRS Editions, 408.

Rosenau P., 1986. Dynamics of nonlinear mass-spring chains near the continuum limit, Physica D: Nonlinear Phenomena $27,224-234$.

Rosenau P., 1987. Quasi-continuous spatial motion of a mass-spring chain, Physics Letters A 118, 222-227.

Scott A. C., 1969. A Nonlinear Klein-Gordon Equation, American Journal of Physics 37, 52-61.

Scott A., 2005. Nonlinear Science, Oxford Texts in Applied and Engineering Mathematics 8, 496.

Smirnov V. V., Manevitch L. I., Strozzi M., Pellicano F., 2016. Nonlinear optical vibrations of single-walled carbon nanotubes. 1. Energy exchange and localization of low-frequency oscillations, Physica D 325, 113-125.

Strozzi M., Smirnov V. V., Manevitch L. I., Milani M., Pellicano F., 2016. Nonlinear vibrations and energy exchange of single-walled carbon nanotubes. Circumferential flexural modes, Journal of Sound and Vibration 381, 156-178.

Ture Savadkoohi A., Lamarque C.-H., Contessa M. V., 2016. Trapping vibratory energy of main linear structures by coupling light systems with geometrical and material non-linearities, International Journal of Non-Linear Mechanics 80, 3-13.

Vakakis A.F., King M. E., Pearlstein A., 1993. Forced localization in a periodic chain of nonlinear oscillators, International Journal of Non-Linear Mechanics 29, 429-447.

Vakakis A.F., Manevitch L.I., Mikhin Y.V., Pilipchuk V.N., Zevin A.A., 1996. Normal Modes and Localization in Nonlinear Systems, John Wiley \& Sons, Inc., New York, 552.

Zakharov V.E., Manakov S.V., Novikov S.P., Pitaevskii L.P., 1980. Theory of solitons: The inverse scattering method, Moscow Nauka (Translation: New York and London, Consultants Bureau, 1984), 286. 